\title{
A Novel Titanium Nitride (TiN) Coating Enhances Early Osseointegration of Titanium Alloy Pins in Rat Femora
}

\author{
G. Sovak*, A. Weiss*, Y. Segev*, I. Gotman**, E. Gotmanas** and R. Coleman* \\ *Rappaport Faculty of Medicine, Technion - Israel Institute of Technology, Haifa 31096, Israel \\ ** Faculty of Material Engineering, Technion - Israel Institute of Technology, Haifa 3200, Israel
}

Objectives: to test the time course of osseointegration of titanium alloy pins coated with TiN by a novel PIRAC method. Introduction: Due to its good osteointegrative properties, high fatigue strength and low elastic modulus, Titanium is the material of choice for cementless total hip replacement [1]. The main disadvantage of titanium is its poor wear resistance [2]. A hard TiN coating may improve the abrasive and adhesive wear of TiN coating by Physical Vapor Deposition (PVD)[2]. In this study we tested TiN coating by a new PIRAC (Powder Immersion Reaction Assisted Coating) deposition method that produces a layer several micrometers thick [3]. Previously we showed that pins coated with TiN by PIRAC underwent good osseointegration in rat femora 2 months after implantation [4]. The aim of the present study was to study the osseointegration using a time course, 3 days to 6 months. Methods: TiN coated and uncoated Titanium alloy, $1 \mathrm{~mm}$ in diameter and $2 \mathrm{~cm}$ long, were inserted into the distal femurs of sixmonth-old female Wistar rats. Animals were killed at intervals from 3 days to 6 months after implantation. Femurs were embedded in LKB Historesin and $1 \mathrm{~mm}$ thick transverse sections were cut with a low-speed diamond saw. SEM was used to evaluate the bone-implant contact. Frozen sections of decalcified specimens were examined for the activity of alkaline phosphatase (ALP) as a marker for osteoblasts, tartrate resistant acid phosphatass (TRAP) as a marker for osteoclasts and non-specific esterase (NSE) as a marker for macrophage activity.

Results: Our findings show that 3 days after implantation there is a strong ALP activity around the implant, although there was still no bone present near the implant (Fig. 1). After 10 days organization of bone trabeculae around implants was seen in the epiphysis, but not in the diaphysis. 6 months after implantation bone trabeculae in contact with the implant were seen in both the epiphysis and diaphysis. At 2 and 6 months the implants were surrounded by a bone collar (Fig. 2). Morphometric analysis of bone-implant contact (BIC) showed an increase of BIC at all intervals, significantly at 1 month (Table 1$)$.

Conclusion: we conclude that the novel TIN-coated Ti alloy implant underwent improved and quicker osseointegration in the rat femora. This coating can reduce implant wear and also can enhance the implant osseointegration in the patient and thus shorten the healing process.

\section{References}

[1] W.C. Head et al., Clin. Orthop. 311 (1995) 85.

[2] E.A. Salvati, et al., Clin. Orthop. 293 (1993) 160.

[3] A. Shenhar, et al., Symposium XI - Materials in Clinical Application, Florence, 1999.

[4] G. Sovak, et al., J Bone Joint Surg [Br]. 82-B (2000) 290.

[5] This research was supported by a grant from the Ministry of Science, Israel. 

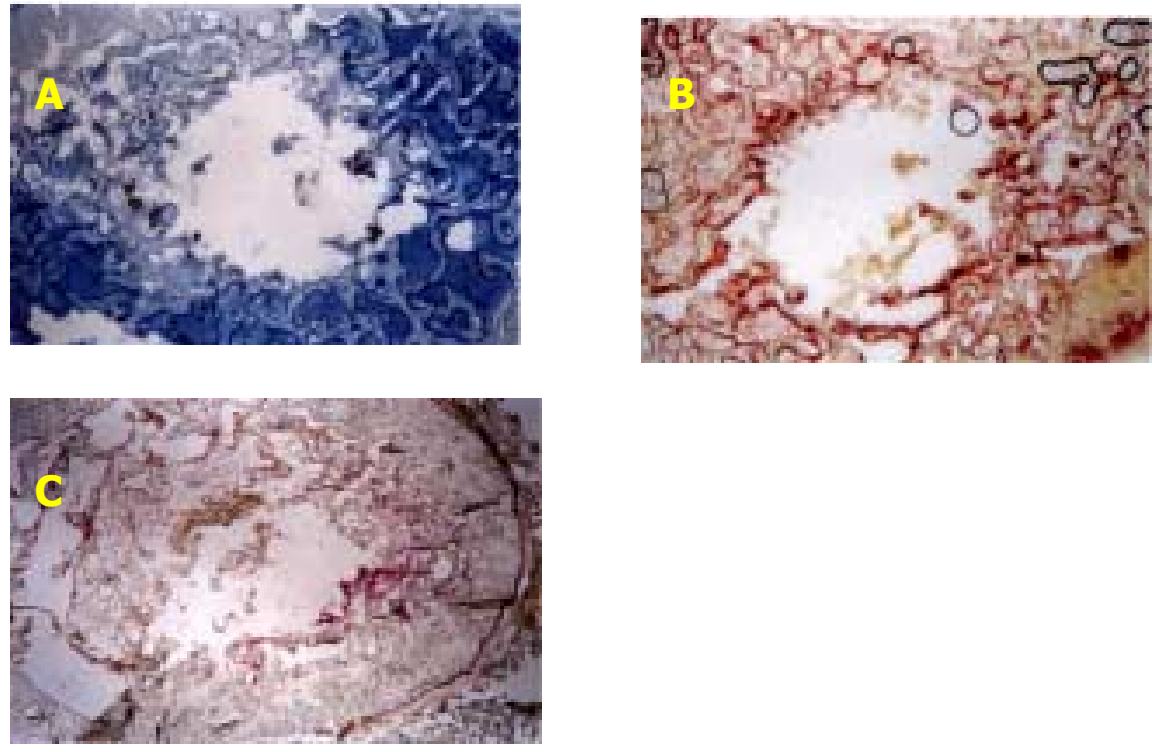

FIG. 1. Histochemical activities in frozen transverse sections of rat femora, with TiN coated implants, 3 days post implantation,. A) NSE as a marker for macrophages, B) ALP as a marker for osteoblasts and C) TRAP as a marker of osteoclasts. Strong activity of all thee enzymes is seen.
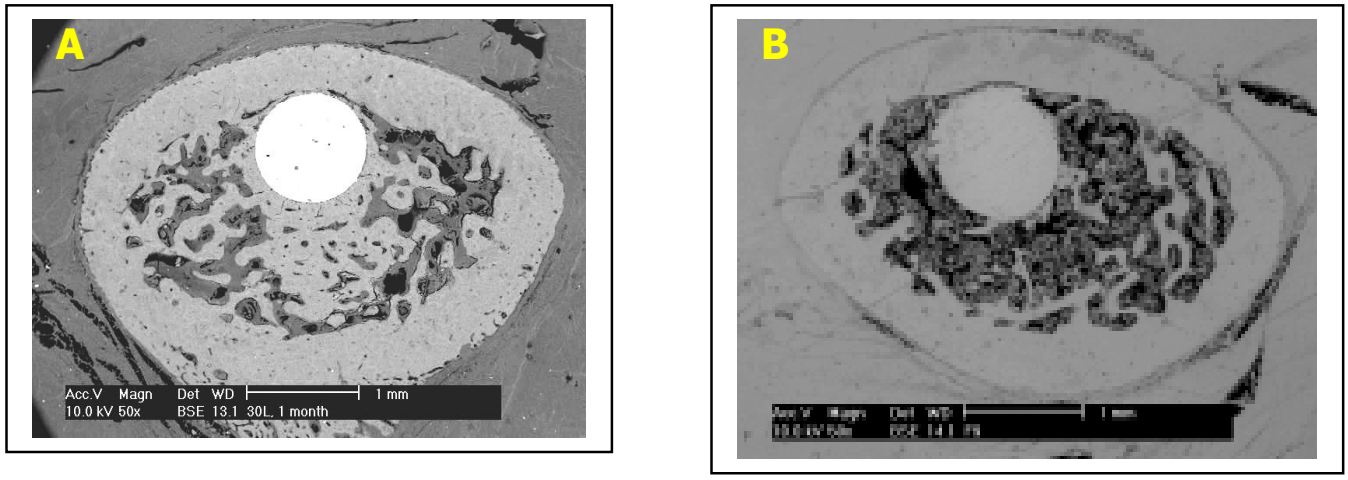

FIG. 2. A SEM microphotograph of a transverse section through the diaphysis 1 month post implantation. A) TiN-coated implant, B) uncoated implant.

TABLE 1. Bone implant contact (BIC)

\begin{tabular}{lll}
\hline & BIC \% & \\
& TiN & Ti \\
\hline 10 days & $75.9 \pm 6.9$ & $65.5 \pm 6.9$ \\
1 month & $82.6 \pm 3.3$ & $54.7 \pm 7.4$ \\
2 months & $88.5 \pm 6.5$ & $81 \pm 5.4$ \\
6 months & -------- & -------- \\
\hline
\end{tabular}

